DRAFT VERSION MAY 26, 2022

Typeset using LATEX twocolumn style in AASTeX62

\title{
SCULPTING ECCENTRIC DEBRIS DISKS WITH ECCENTRIC GAS RINGS
}

\author{
JoNATHAN W. Lin ${ }^{1,2}$ AND EugEne ChiAnG ${ }^{1,3}$ \\ ${ }^{1}$ Department of Astronomy, University of California Berkeley, Berkeley, CA 94720-3411, USA \\ ${ }^{2}$ Department of Engineering Science, University of California Berkeley, Berkeley, CA 94720-1702, USA \\ ${ }^{3}$ Department of Earth and Planetary Science, University of California Berkeley, Berkeley, CA 94720-4767, USA
}

\begin{abstract}
Many debris disks seen in scattered light have shapes that imply their dust grains trace highly eccentric, apsidally aligned orbits. Apsidal alignment is surprising, especially for dust. Even when born from an apse-aligned ring of parent bodies, dust grains have their periastra dispersed in all directions by stellar radiation pressure. The periastra cannot be re-oriented by planets within the short dust lifetimes at the bottom of the collisional cascade. We propose that what re-aligns dust orbits is drag exerted by second-generation gas. Gas is largely immune to radiation pressure, and when released by photodesorption or collisions within an eccentric ring of parent bodies should occupy a similarly eccentric, apse-aligned ring. Dust grains launched onto misaligned orbits cross the eccentric gas ring supersonically and can become dragged into alignment within collisional lifetimes. The resultant dust configurations, viewed nearly but not exactly edge-on, with periastra pointing away from the observer, appear moth-like, with kinked wings and even doubled pairs of wings, explaining otherwise mysterious features in HD 61005 ("The Moth") and HD 32297, including their central bulbs when we account for strong forward scattering from irregularly shaped particles. Around these systems we predict gas at Kuiper-belt-like distances to move on highly elliptical streamlines that owe their elongation, ultimately, to highly eccentric planets. Unresolved issues and an alternative explanation for apsidal alignment are outlined.
\end{abstract}

Keywords: interplanetary medium, zodiacal dust, planet-disk interactions, stars: individual (HD 32297, HD 61005)

\section{INTRODUCTION}

Debris disks are composed of optically thin dust generated from the collisional destruction of larger parent bodies. They exhibit diverse morphologies; see Schneider et al. (2014) for an atlas of disk images in scattered starlight. The various non-axisymmetric shapes have been interpreted by Lee \& Chiang (2016, LC16) in terms of a narrow, possibly eccentric ring of parent bodies, shaped gravitationally by a neighboring planet, grinding into dust particles whose orbits are widened by stellar radiation pressure. The model provides a framework for understanding a wide array of observed disk structures that have been likened to, e.g., "needles," "fans," "bars," and "ship wakes," all by simple changes in viewing geometry.

Dust grain orbits have a large range of semimajor axes and eccentricities, with apastra tending to infinity (in vacuum) as grains approach the radiation blow-out size. A major factor in determining the appearance of a debris disk is the extent

Corresponding author: Jonathan W. Lin

jonathan18808@gmail.com, echiang@astro.berkeley.edu to which dust particle orbits are apsidally aligned, i.e., the degree to which their periastra point in the same direction. Apsidal alignment maximizes non-axisymmetry. According to LC16, alignment is needed to explain two features related to the "moth"-like shape that results when a highly eccentric ( $e \gtrsim 0.5$ ) parent body ring is viewed nearly but not completely edge-on, with apastron directed out of the sky plane toward the observer. ${ }^{1}$ The first aspect of the moth necessitating apsidal alignment is the angle at which the tips of the moth wings sweep away from the horizontal. In HD 61005 (the eponymous "Moth"; Hines et al. 2007; Maness et al. 2009; Ricarte et al. 2013), the wingtips are canted by $\sim 23^{\circ}$ (Buenzli et al. 2010; Esposito et al. 2016). Such a large, order-unity "kink" from the horizontal (Esposito et al. 2016 refer to this feature as an "elbow") cannot be reproduced if dust grain orbits are apsidally misaligned; the resultant density distribution is too axially symmetric and the isophotes too rounded (LC16, their Figure 6). The second feature that

\footnotetext{
${ }^{1}$ When the apsidal line is in the sky plane, the disk resembles a lopsided "needle", as in HD 15115 (LC16).
} 
hinges on apsidal alignment is the "double wing"- a secondary band of light which is offset from the star and nearly parallel to the primary band (LC16, their Figure 9). The secondary wing traces overdensities that are peculiar to perfectly apsidally aligned orbits whose apastra span a large range (LC16, their Figure 7; we recapitulate their explanation in $\S 3$ of our paper). The debris disk HD 32297 is a double-winged moth (see Figures 18 and 19 of Schneider et al. 2014) as is HD 61005 (see Figure 7 of Schneider et al. 2014; Esposito et al. 2016 refer to the multiple radial extensions as "spurs").

Apsidal alignment of dust grain orbits is surprising. Dust particles are not apsidally aligned when launched from an apse-aligned ring of parent bodies; stellar radiation pressure disperses dust grain longitudes of periastra so that they span the full gamut between 0 and $2 \pi$ (e.g., equation 10 of LC16). The apsidally aligned configurations modeled by LC16 were assumed without justification; they resulted from demanding that dust-producing collisions between parent bodies occur strictly at periastron of the parent ring (their "periapse only" scenario; see also Esposito et al. 2016 who made the same assumption). But restricting collisions to periastron only is unphysical; relative velocities and densities of parent bodies can vary only smoothly and quasi-sinusoidally with orbital phase, with collisions happening everywhere. If, more realistically, we launch dust grains using a uniform distribution of parent body mean anomalies, or a uniform distribution of parent body true anomalies (for more discussion of these distributions, see $\S 3$ ), the kink and double wing disappear (LC16 Figure 6). Moreover, we cannot look to any planet to align dust grain orbits, as forced precession timescales are typically much longer than the short collisional lifetimes of the small dust particles that dominate scattered light images.

Thus how to achieve the strong alignment demanded by observations has been a problem. There is, however, another player that has yet to enter the field. Gas may be present in debris disks, released by volatile-rich solids by photodesorption (e.g., Chen et al. 2007; Grigorieva et al. 2007) or vaporization following collisions (e.g., Czechowski \& Mann 2007). The Atacama Large Millimeter Array and the Herschel Space Observatory have detected $\mathrm{CO}$ and its photodissociation products $\mathrm{C}$ and $\mathrm{O}$ around a slew of debris disks (e.g., Kral et al. 2017; Matrà et al. 2019). The growing list includes the double-winged moth HD 32297, around which a CO mass of $\sim 4 \times 10^{-4} M_{\oplus}$ (MacGregor et al. 2018) and a neutral C mass of $\sim 3.5 \times 10^{-3} M_{\oplus}$ (Cataldi et al. 2019) have been inferred. The aforementioned $\mathrm{CO}$ mass is a lower limit because it is based on observations of ${ }^{12} \mathrm{CO}$ gas which may be optically thick; Moór et al. (2019, submitted) report up to $\sim 0.07 M_{\oplus}$ using trace $\mathrm{CO}$ isotopologues. At the same time, $\mathrm{CO}$ masses derived assuming local thermodynamic equilibrium (LTE) may be overestimated if radiative effects drive the line excitation temperature above the gas kinetic temperature (e.g., Matrà et al. 2015; Cavallius et al. 2019). In the case of HD 61005, neither CO (Olofsson et al. 2016; MacGregor et al. 2018) nor neutral O (Riviere-Marichalar et al. 2016) has been detected. While there may be less than $7.7 \times 10^{-7} M_{\oplus}$ in CO (derived assuming optically thin LTE and therefore subject to revision), current limits on the atomic gas mass in HD 61005 might not be as severe. A crude comparison point is provided by $\beta$ Pic, for which O I was detected at $5 \sigma$ (Brandeker et al. 2016) and $\mathrm{C}$ I detected at $8 \sigma$, implying a neutral $\mathrm{C}$ mass between $5 \times 10^{-4}$ and $3 \times 10^{-3} M_{\oplus}$ (Cataldi et al. 2018). As $\beta$ Pic is closer to Earth than HD 61005 (19.4 vs. 35.3 pc; e.g., Matrà et al. 2018), an atomic disk of similar mass, say $10^{-3} M_{\oplus}$, might also orbit HD 61005 and marginally elude detection by Herschel (assuming the two systems have similar line excitation conditions powered by the ultraviolet interstellar radiation field; e.g., Kral et al. 2016).

Here we consider the effects of gas drag on debris disk dust, with an eye toward explaining apsidally aligned systems like the Moth and HD 32297. We focus on the case where gas is generated by an eccentric, apse-aligned ring of parent bodies; it is within the parent ring that particle densities and by extension collision/vaporization rates are highest. At least initially upon release, such gas should be co-located with the parent ring, occupying similarly eccentric and apsealigned orbits; gas is released from solids with the latter's orbital velocities and at all stages of the collisional cascade, ${ }^{2}$ and gas does not, for the most part, feel radiation pressure (Fernández et al. 2006; Roberge et al. 2006; Kral et al. 2017). By contrast, dust does feel radiation pressure, and will intersect the gas on different, apsidally misaligned trajectories. The question is whether the gas can bring dust grain orbits into alignment.

In this paper we consider a simplified version of this problem, idealizing what in reality is a radially distributed gas disk with a single elliptical ring of zero width. This simplification, though drastic, helps us acquire intuition, avoids the introduction of unconstrained parameters, and promises to yield results that are correct to order-of-magnitude insofar as most of the torque on a dust grain may be exerted at a particular orbital radius (depending on density and eccentricity gradients in the gas). After solving for individual dust particle trajectories in $\S 2$, we synthesize full scattered light

\footnotetext{
2 There is a complication here, and that is when gas is released predominantly from the smallest bound dust grains at the bottom of the collisional cascade. This is a possibility insofar as such grains dominate the collective surface area. Since these grains feel radiation pressure and are not apse-aligned, the gas they generate may also not be apse-aligned. On the other hand, like all other bodies, these grains have their highest densities and therefore produce the most gas within the parent ring where they were born, where their orbits converge, and where their local trajectories are more similar to those of the apse-aligned parents. Within this dense region, collisions between gas molecules will average out their velocities and may bring the gas back into apsidal alignment.
} 
images in $\S 3$ to see if we can reproduce the double-winged moth. A summary and discussion of model limitations and unresolved issues, including mention of an alternative scenario to generate apse-aligned orbits, are given in $\S 4$.

\section{SCULPTING OF DUST GRAIN ORBITS BY AN ELLIPTICAL GAS RING}

Consider a dust grain on an elliptical orbit intersecting an elliptical ring of gas. Co-planar, apsidally misaligned orbits can cross each other twice. At each crossing, the gas-dust relative velocity $\Delta \vec{v}_{\text {rel }} \equiv \vec{v}_{\text {gas }}-\vec{v}_{\text {dust }}$ can be large, having a magnitude $\Delta v_{\text {rel }}=\left|\Delta \vec{v}_{\text {rel }}\right|$ comparable to the local Keplerian velocity $v_{\mathrm{K}}$. At radial distances $r \sim 100 \mathrm{AU}$ from a star of mass $M_{*} \sim 1 M_{\odot}, v_{\mathrm{K}} \sim 3 \mathrm{~km} / \mathrm{s}$. This exceeds the sound speed $c_{\mathrm{s}} \sim 0.2(T / 60 \mathrm{~K})^{1 / 2}(14 \mathrm{amu} / \mu)^{1 / 2} \mathrm{~km} / \mathrm{s}$ in gas of mean molecular weight $\mu$ and temperature $T$ (see Kral et al. 2016 for possible gas properties). Thus $\Delta v_{\text {rel }}$ can be supersonic, in which case the force of gas drag on the grain is quadratic in $\Delta v_{\text {rel }}$ (not linear as in subsonic Epstein drag):

$$
\vec{F}_{\text {drag }}=\frac{1}{2} C_{\mathrm{D}} \rho_{\text {gas }} \Delta v_{\text {rel }} \Delta \vec{v}_{\text {rel }} \pi s^{2}
$$

where $\rho_{\text {gas }}$ is the gas density, $s$ is the grain radius, and $C_{\mathrm{D}}$ is a dimensionless drag coefficient (e.g., Munson et al. 2006).

Each time a dust grain of mass $m_{\text {dust }}=(4 / 3) \pi \rho_{\mathrm{p}} s^{3}$ and internal density $\rho_{\mathrm{p}}$ crosses the gas ring, the grain velocity changes by

$$
\Delta \vec{v}_{\text {kick }}=\frac{\vec{F}_{\text {drag }}}{m_{\text {dust }}} \Delta t \sim \frac{\vec{F}_{\text {drag }}}{m_{\text {dust }}} \frac{\Delta \ell}{v_{\mathrm{K}}}
$$

where $\Delta t \sim \Delta \ell / v_{\mathrm{K}}$ is the time it takes the particle to cross the ring of local span $\Delta \ell$. Neglecting details of geometry (e.g., width variations as a function of azimuth, and density gradients), we approximate the gas ring mass as $M_{\text {gas }} \sim$ $2 \pi r \Delta r H \rho_{\text {gas }}$, where $\Delta r$ and $H$ are characteristic radial and vertical ring thicknesses. Then

$$
\Delta \vec{v}_{\text {kick }}=A \Delta v_{\text {rel }} \Delta \vec{v}_{\text {rel }}
$$

where

$$
\begin{gathered}
A \sim 0.04\left(\frac{C_{\mathrm{D}}}{1}\right)\left(\frac{M_{\mathrm{gas}}}{10^{-3} M_{\oplus}}\right)\left(\frac{1 \mathrm{~g} / \mathrm{cc}}{\rho_{\mathrm{p}}}\right)\left(\frac{0.5 \mu \mathrm{m}}{s}\right) \times \\
\left(\frac{M_{\odot}}{M_{*}}\right)^{1 / 2}\left(\frac{40 \mathrm{AU}}{r}\right)^{3 / 2}\left(\frac{0.1}{H / r}\right)\left(\frac{\Delta \ell / \Delta r}{1}\right) \mathrm{s} / \mathrm{km} .
\end{gathered}
$$

We have normalized equation (4) using a total gas mass of $M_{\text {gas }}=10^{-3} M_{\oplus}$, a value that seems within the (admittedly highly uncertain) range of possible gas masses in HD 32297 and HD 61005 ( $(1)$. Other values of $A$ are considered in the last paragraph of $\S 3$.
Each ring crossing changes the velocity of the dust grain relative to the gas by a fractional amount

$$
\frac{\Delta v_{\text {kick }}}{\Delta v_{\text {rel }}} \sim 0.1\left(\frac{A}{0.04 \mathrm{~s} / \mathrm{km}}\right)\left(\frac{\Delta v_{\text {rel }}}{3 \mathrm{~km} / \mathrm{s}}\right) .
$$

Thus for our nominal parameters it takes on the order of 10 orbits for the dust particle's velocity to relax, locally, to that of the gas ring (we will shortly obtain more precise estimates of the orbit convergence time by numerical integration). It should be checked that while the dust grain's orbit is evolving from gas drag, the grain is safe from collisional destruction by other grains. Dust optical depths $\tau$ measured in the radial direction in HD 61005 and HD 32297 are $\sim 10^{-2}$ (using equation 7 of Chiang et al. 2009), and so a grain at the bottom of the collisional cascade (these dominate the scattering optical depth) will execute $\sim 1 / \tau \sim 10^{2}$ orbits before colliding with another grain and shattering to blow-out size.

Figure 1 illustrates the effects of gas drag on dust grain orbits. We launch dust particles from an elliptical parent body ring over a range of parent body true anomalies $f_{\mathrm{p}}$; the initial velocity of a particle is identical to the parent body velocity at the launch point. The gas ring is idealized as a single fixed ellipse that is co-planar and apse-aligned with the parent body ring. While the rings are assumed to have similar eccentricities, the gas ring is situated outside the parent body ring so that a dust grain launched from the latter may intersect the former with non-zero relative velocity. At the intersection point, a velocity impulse due to gas drag is applied to change the dust orbit instantaneously. Had we placed the gas ring interior to the parent body ring, dust grains launched from the parent ring would never intersect the gas ring, because radiation pressure forces dust grain orbits to always lie outside the parent body ring. Had we overlapped the gas and parent body rings completely, dust-gas relative velocities and hence drag would be zero because, although dust and gas have different orbits because of radiation pressure, their orbits coincide perfectly at the parent ring from where they are both launched (with the same parent body velocity). These same statements apply when we consider the more realistic case that gas is radially distributed: a given grain will only cross the orbits of gas lying exterior to where the grain was born. To this extent our placement of the zero-width gas ring exterior to the parent body ring is realistic. What is not so realistic is our simplifying assumption of zero width; our reliance on the impulse approximation means we cannot model gas disks that are too massive and that change a dust grain's orbit gradually over large distances. We will hit against this limitation at the end of $\S 3$, and reiterate it in the context of observations in $\S 4$.

Setting $A=0.04 \mathrm{~s} / \mathrm{km}$ in equation (3) constant, we apply a velocity impulse $\Delta \vec{v}_{\text {kick }}$ to a dust particle each time it crosses the gas ring, after which we update the Keplerian el- 
ements of the dust orbit and solve for the location of the next gas ring crossing. We plot dust grain orbits after $\eta_{\text {kick }}=10^{2}$ gas ring crossings $=50$ orbital periods, choosing each particle's $\beta$-the force ratio between stellar radiation pressure and gravity - to be as large as possible while keeping the particle bound (such high- $\beta$ particles dominate optical depths because they reside on large orbits with long collisional lifetimes). As Figure 1 shows, when gas ring/parent body eccentricities are as large as $0.5-0.7$ (top panels), dust particles have their apsidal lines dragged into close alignment with the gas ring. Most initial conditions (launch true anomalies $f_{\mathrm{p}}$ ) result in apsidal alignment; only when $f_{\mathrm{p}}$ is near $\pi$ does the particle remain on a nearly anti-aligned orbit (with particle and gas periastra nearly $180^{\circ}$ apart). When parent body and gas ring eccentricities are reduced to $\sim 0.2-0.3$, apsidal lines remain out of alignment (bottom panels of Figure 1).

We present in Figure 2 an example time history of how a particle orbit evolves in the case of high parent body/gas ring eccentricity. Orbital contraction is followed by expansion; since the gas velocity can exceed the particle velocity at the location of ring crossing, gas drag does not necessarily reduce the particle's orbital energy. In fact, under some circumstances, dust particles can receive so much energy from gas that, in combination with stellar radiation pressure, particles become unbound (see also §3). In general, gas drag tends to erase velocity differences between a particle and the gas ring, slaving the former to the latter so that their apsidal lines eventually coincide. The apsidal line precesses as the impulses imparted at one ring crossing outweigh those at the other; the crossing closer to gas periapse tends to dominate insofar as $\Delta v_{\text {rel }}$ is higher there. For our nominal parameters, it takes a few dozen gas ring crossings for the two crossing points to converge toward gas periapse (see orbit 4 in Figure 2) and for the dust grain orbit to approach its final shape. This alignment timescale is shorter, albeit only marginally, than the grain-grain collisional lifetime, estimated above to be on the order of $10^{2}$ orbits. With the particle and gas velocities nearly equal at gas periapse, the particle traces a larger orbit relative to gas because the former feels a gravitational potential that is weakened by $1-\beta$. We call this final dust orbit an "attractor orbit"; we will see such orbits manifest in scattered light images.

\section{SCATTERED LIGHT IMAGES}

Our procedure for generating synthetic scattered light images largely follows that of LC16. The main difference is that we evolve dust grain orbits by gas drag, in similar fashion to the examples shown in Figures 1 and 2. The model ingredients and recipe for combining them are as follows (for details, see LC16): (i) A planet of mass $10 M_{\oplus}$ resides on an orbit of semimajor axis $a_{\text {planet }}=30 \mathrm{AU}$ and eccentricity $e_{\text {planet }}=0.7$ about a star of mass $M_{*}=1 M_{\odot}$.

(ii) $N_{\mathrm{p}}=1000$ parent bodies are randomly drawn from an elliptical annulus whose semimajor axes extend from $a_{\mathrm{p} \text {,inner }}=33 \mathrm{AU}$ (just outside the planet's chaotic zone) to $a_{\mathrm{p}, \text { outer }}=43 \mathrm{AU}$ (fractional width $30 \%$ ).

(iii) The forced eccentricity vectors of the parent bodies are given by Laplace-Lagrange secular theory, and their free eccentricity vectors are randomly oriented and range up to 0.02 in length. As such, the parent body orbits have eccentricities of $0.5-0.7$, and are on average apsidally aligned with the planet's orbit. Mutual inclinations between all bodies are set to zero for simplicity (this choice differs from LC16).

(iv) Along each parent body orbit, we randomly draw $N_{\text {launch }}=10^{2}$ locations for launching dust grains. We experiment with two ways of drawing these launch sites; either we draw parent body mean anomalies $M_{\mathrm{p}}$ uniformly between 0 and $2 \pi$, or we draw parent body true anomalies $f_{\mathrm{p}}$ uniformly between 0 and $2 \pi$. The former concentrates collisions near apoapse, and generates initial dust orbits that are not apsidally aligned (see LC16 Figure 6, bottom row); by contrast, the latter concentrates collisions near periapse, and generates initial dust orbits that exhibit some preference for being apsidally aligned (even without gas drag). Reality is presumably bracketed by these two cases. ${ }^{3}$ At every $M_{\mathrm{p}}$ or $f_{\mathrm{p}}$, a dust particle orbit is created whose velocity at that position matches the parent body's, and whose $\beta$ is drawn from a Dohnanyi size distribution modified for the long collisional lifetimes at high $\beta$ (see equations 8-14 of LC16).

(v) Each dust grain orbit is modified by applying velocity impulses at $\eta_{\text {kick }}=10^{2}$ gas ring crossings. Impulses are evaluated according to equation (3) at fixed $A=0.04 \mathrm{~s} / \mathrm{km}$. The gas ring is idealized as a single ellipse of eccentricity 0.56 and semimajor axis $44 \mathrm{AU}$, apse-aligned with the planet and nearly coincident with the outermost parent body orbit. ${ }^{4}$

\footnotetext{
${ }^{3}$ The distribution of launch sites follows the grain-grain collision rate, which scales as the relative grain-grain velocity times the square of the grain density. Relative velocities, insofar as they scale with the mean orbital velocity, are higher near periapse; densities measured per unit azimuthal distance are higher near apoapse because particles spend more time there; and densities measured per unit radial distance are higher at either peri or apo depending on how parent body eccentricities vary with semimajor axis, i.e., whether $d e / d a$ is positive or negative. This last factor depends on whether the perturber is interior or exterior to the ring. Rather than micro-model these details, our simple mean vs. true anomaly prescriptions try to circumscribe the range of possibilities; the details are not as important to capture as the smooth and quasi-sinusoidal variation of the collision rate with azimuth.

${ }^{4}$ Technically, the gas ring semi-major axis is chosen to be $2 \%$ larger than that of the outermost parent body orbit, to ensure that no dust grain orbit lies completely exterior to the gas ring; see our discussion of the placement of the gas ring in $\S 2$. Dust grain orbits that lie completely interior to the gas ring are not modified by gas drag. These represent low-eccentricity, low- $\beta$ particles that, were we to model a more realistic gas ring of finite width,
} 


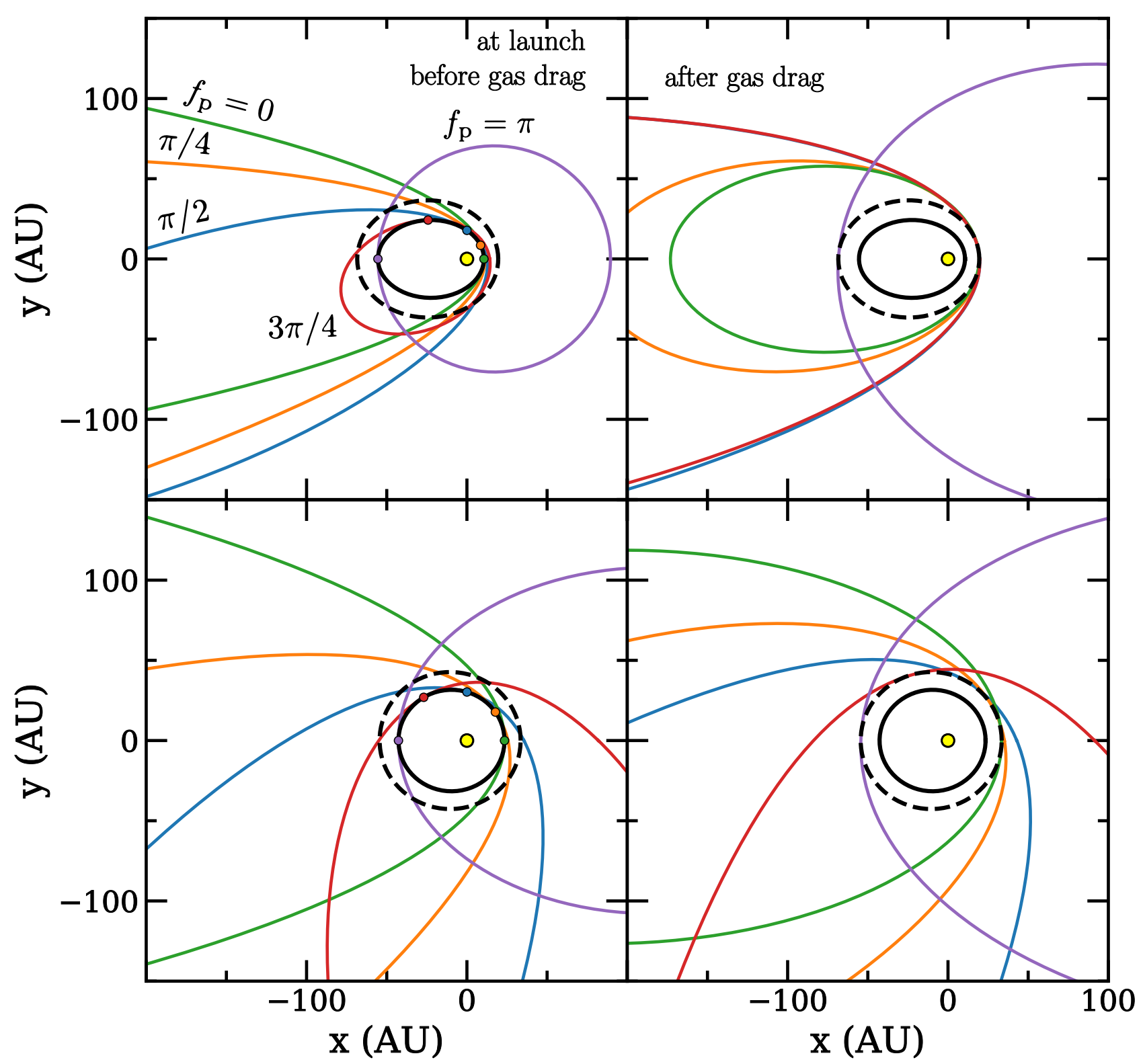

Figure 1. Torquing dust grain orbits by gas drag. Left panels: Dust grains are launched from a parent body orbit (black solid ellipse) whose semi-major axis $a_{\mathrm{p}}=33 \mathrm{AU}$ and eccentricity $e_{\mathrm{p}}=0.68$ (top) or 0.29 (bottom). Launch points are denoted by circles colored the same as corresponding dust orbits, with parent body true anomalies $f_{\mathrm{p}}$ at launch annotated. For each dust grain, the force ratio $\beta$ between radiation pressure and gravity is maximized while ensuring the grain is not ejected after gas drag is applied. The gas ring, idealized as a single orbit (black dashed ellipse), has semi-major axis $44 \mathrm{AU}$ and eccentricity 0.56 (top) or 0.24 (bottom); the gas ring eccentricity is slightly lower than the eccentricity of the parent body ring because both eccentricities are imagined to be forced by a planet (not shown), which lying interior to the parent body ring is farther from the gas ring. For high $e_{\mathrm{p}}$, initial dust orbits are already somewhat apsidally aligned (see also Figure 5 of LC16 and related discussion) but not enough to reproduce the kink or double wing in scattered light images (see Figures 3-6). Right panels: Dust grain orbits after $\eta_{\text {kick }}=100$ gas ring crossings, with fixed gas-drag impulse coefficient $A=0.04$ s/km (see equation 3). Applying more kicks than $\eta_{\text {kick }}=100$ produces no further changes in the dust orbits. For high $e_{\mathrm{p}}$ (top panels), dust grain orbits (excepting the one launched at $f_{\mathrm{p}}=\pi$ ) become strongly apsidally aligned. For low $e_{\mathrm{p}}$ (bottom panels), alignment remains negligible. Apsidal alignment is easiest to achieve at high parent body and gas ring eccentricity. 


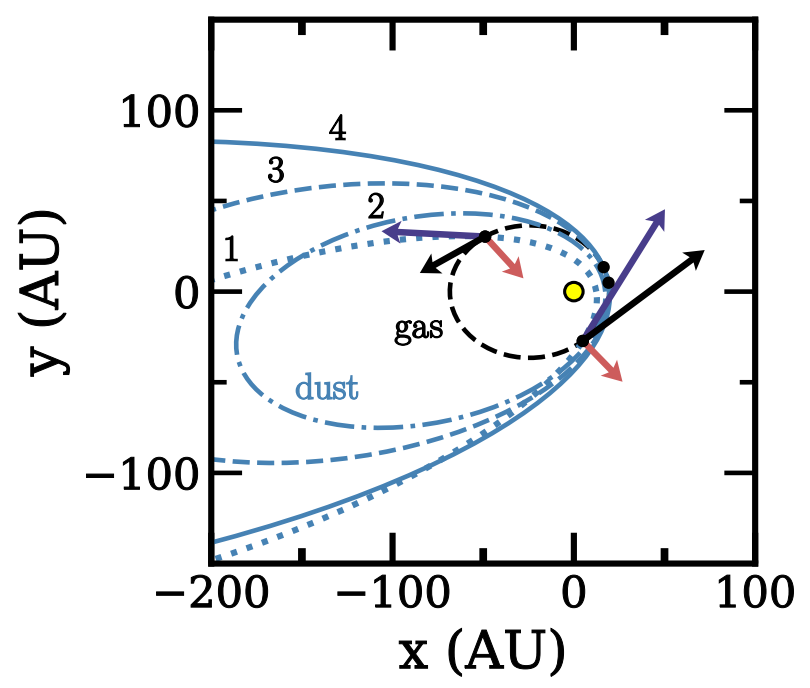

Figure 2. Time evolution of a dust grain orbit (blue) intersecting a gas ring (dashed black). The grain is identical to the one in the top panels of Figure 1 (shown also in blue), launched at $f_{\mathrm{p}}=\pi / 2$ from a parent body of eccentricity $e_{\mathrm{p}}=0.68$. The time sequence $\{1,2,3,4\}$ corresponds to $\{0,10,35,85\}$ gas ring crossings, computed for $A=0.04 \mathrm{~s} / \mathrm{km}$. For orbit 1 , the locations where the dust grain orbit and gas ring cross are shown as black dots, together with the local gas velocity vector (black), dust velocity (blue), and the gasdust relative velocity $\Delta \vec{v}_{\text {rel }}$ (red); these vectors are drawn to scale and demonstrate that the gas-dust relative velocity can be comparable to the local, supersonic orbital velocity. Gas ring crossings are also marked on orbit 4; these have converged toward gas periapse as dust becomes apsidally aligned with gas.

At every crossing of the gas ring, the Keplerian elements of a dust particle orbit are updated and the location of the next ring crossing computed. Dust grain orbits that become unbound are discarded; about half are lost this way (somewhat more in the case of uniform $M_{\mathrm{p}}$ ).

(vi) Along every dust orbit we lay down $N_{\text {dust-per-orbit }}=$ $10^{2}$ dust particles with mean anomalies randomly drawn from a uniform distribution. If gas drag had not eliminated some orbits, we would have drawn $N_{\mathrm{d}}=N_{\mathrm{p}} \times N_{\text {launch }} \times$ $N_{\text {dust-per-orbit }}=10^{7}$ dust particles; in practice, step (v) reduces $N_{\mathrm{d}}$ to $3-5.5 \times 10^{6}$. Surviving grains are projected onto the sky plane of a distant observer to create a scattered light image. We experiment with two scattering phase functions (SPFs): a Henyey-Greenstein function with asymmetry parameter $g=0.5$, and the SPF inferred by Hed-

would be wholly immersed in gas. In the limit of small $\beta$, their orbits would hardly change, aside from a slow radial drift. man \& Stark (2015) for Saturn's irregularly shaped, strongly forward-scattering ring particles. Images are smoothed with a 2D Gaussian having a standard deviation of $1 \mathrm{AU}$, and scaled to the square root of the surface brightness to bring out fainter features.

Step (v) distinguishes our work from LC16. Our nominal choice for $A=0.04 \mathrm{~s} / \mathrm{km}$ is consistent with grains of roughly micron size interacting with a gas ring of mass $10^{-3} M_{\oplus}$ located around $40 \mathrm{AU}$ (see equation 4 ). The number $\eta_{\text {kick }}=10^{2}$ of velocity impulses applied to each grain (occurring over $\eta / 2=50$ dust grain orbital periods because there are 2 ring crossings per orbit) falls marginally within typical collisional lifetimes in HD 61005 and HD 32297 (§2). We could tailor $A$ for each particle size (equivalently $\beta$ ), but the added realism does not seem worth it, since those particles that dominate the optical depth lie at the bottom of the collisional cascade and span only a factor of $\sim 2$ in size. Other choices for $A$ and $\eta_{\text {kick }}$ are discussed at the end of this section.

Figures 3 and 4 compare disk images with and without sculpting from an elliptical gas ring, for the case where dust orbits are initialized using a uniform distribution of parent body mean anomalies $M_{\mathrm{p}}$. Figures 5 and 6 are the same but for the case where parent body true anomalies $f_{\mathrm{p}}$ are uniformly distributed. We choose a viewing azimuth of $a z=0^{\circ}$ so that apoapse of the parent ring points toward the observer, and an altitude of alt $=-3^{\circ}$ so that the observer views the disk nearly edge-on and from below the disk midplane. Similar viewing angles in LC16 yielded a "moth" with a "double wing". But whereas LC16 assumed that dust grain orbits were apsidally aligned by launching them only at parent body periastra (their "periapse only", $f_{\mathrm{p}}=M_{\mathrm{p}}=0$ case), our model does not make this (unrealistic) assumption; we launch grains over all azimuths from 0 to $2 \pi$, and compute how orbits precess and more generally have their semimajor axes and eccentricities altered by gas drag.

Without gas drag, dust grain apsides are not well aligned (Figure 4 or 6, left) and the resultant moth-like shape (a.k.a. "fan") is rounded and lacks a double wing (Figure 3 or 5, left). With gas drag, dust grain orbits come into much better alignment (Figures 4 and 6, middle); the resultant moth exhibits kinked wings, and a second pair of wings above the first (Figures 3 and 5, middle and right). The kink and the double wing are features reminiscent of those observed in HD 61005 and HD 32297. We are especially encouraged by the disk images generated using the Hedman-Stark SPF; these exhibit a central "bulb" remarkably similar to those observed in HD 32297 and HD 61005-compare the right panels of our Figures 3 and 5 with Figures 19 and 21 of Schneider et al. (2014).

The origin of the double wing is clearer in a face-on $\left(a l t=90^{\circ}\right)$ view, as provided in the rightmost panels of Fig- 


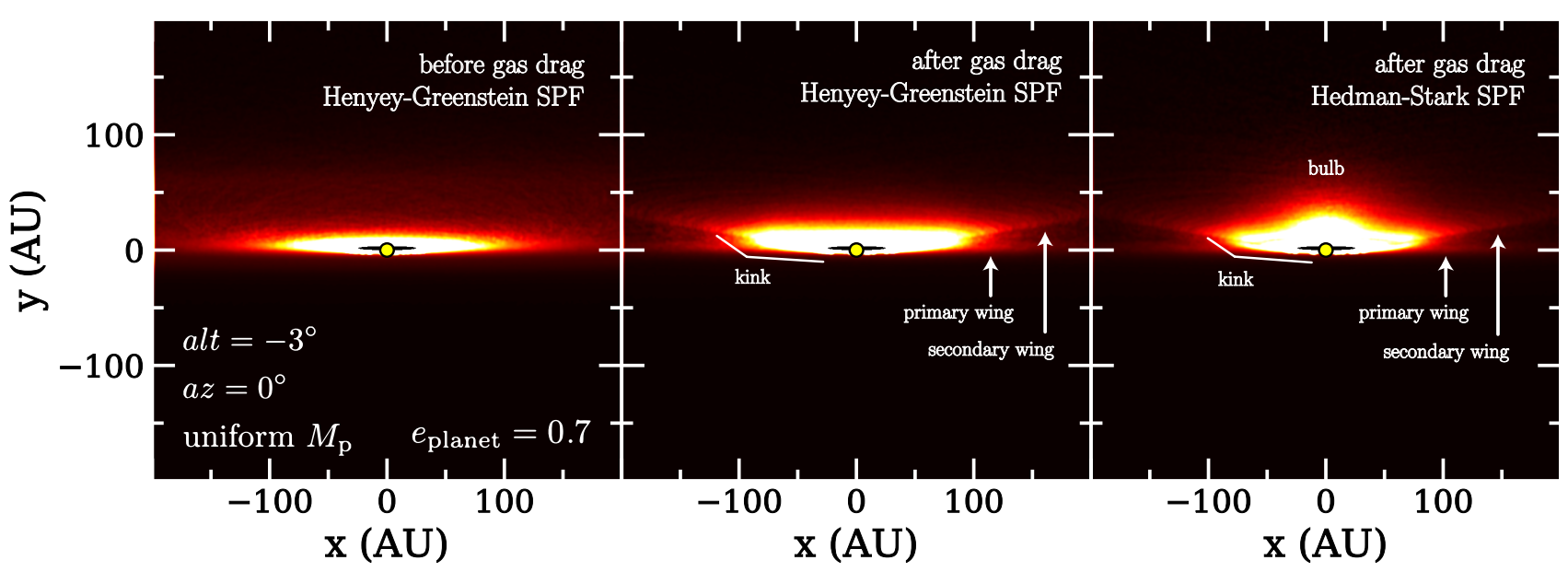

Figure 3. Synthetic scattered light images of a debris disk, before and after dust particles experience gas drag, for the case where dust grains are launched from a uniform distribution of parent body mean anomalies $M_{\mathrm{p}}$. The gas ring nearly coincides with a narrow ring of parent bodies made elliptical by a planet of eccentricity $e_{\text {planet }}=0.7$. The disk is viewed from below its midplane, at an angle alt $=-3^{\circ}$ from edge-on, and at an azimuth $a z=0^{\circ}$ such that apoapse of the parent body ring points toward the observer. Without gas drag (left panel), the isophotes of the moth-like image are rounded because dust grain apsides are not well aligned (see also left panel of Figure 4). With gas drag (applied using $A=0.04 \mathrm{~s} / \mathrm{km}$ and $\eta_{\text {kick }}=10^{2}$ ), many dust grain apsides are forced into much better alignment (Figure 4, middle); a "kink" appears in the wingtips, in addition to a "double wing". These features, which resemble those observed in HD 61005 (e.g., Esposito et al. 2016 ) and HD 32297 (e.g., Schneider et al. 2014), manifest whether we employ a Henyey-Greenstein SPF with $g=0.5$ (middle panel) or a Hedman \& Stark (2015) SPF derived from Saturn's rings (right panel). However, using the Hedman-Stark SPF generates a bulbous top reminiscent of those seen in HD 32297 (see panels B and C of Figure 19 of Schneider et al. 2014) and HD 61005 (see Figure 21 of Schneider et al. 2014); the "bulb" traces dust at the apastra of attractor orbits (Figure 4, right) made visible by strong forward scattering. The images are constructed following nearly the same procedure as in LC16 (in particular they are displayed using the same square root scaling); here the 2D Gaussian used to smooth the images has a standard deviation of $1 \mathrm{AU}$.

ures 4 and 6 , where the secondary wing manifests as two overdense "rays" pointing away from the star. As explained in LC16, the rays arise from two competing effects: the first concentrates particles near their apastra because they spend more time there, and the second dilutes particles along the apsidal line as apastron distances telescope outward as part of a $\beta$-distribution that rises toward blow-out. The net effect is to concentrate particles at orbital phases that are displaced slightly away from apastron. The rays materialize only when orbits are apsidally well-aligned. Comparing our Figures 4 and 6 with Figure 7 of LC16, we see that the main difference in model outcomes is that the (unphysical) periapseonly scenario of LC16 yields orbits having a continuous and smoothly varying range of semimajor axes, whereas our gas ring model selects for certain "attractor orbits" (see discussion at end of §2). Dust grains at the apastra of attractor orbits make up the central bulbs seen in the right panels of Figures 3 and 5; these grains forward-scatter light more strongly at scattering angles of a few degrees using the Hedman-Stark SPF than the Henyey-Greenstein SPF (see Figure 15 of Hedman \& Stark 2015).

The double wing is more easily seen when dust grains are launched with uniform $M_{\mathrm{p}}$ (Figure 3) than with uniform $f_{\mathrm{p}}$ (Figure 5). Ironically this is because the former case yields more dust orbits that are not apsidally aligned (Figure 4, middle; contrast with Figure 6, middle). For uniform $M_{\mathrm{p}}$, the non-aligned population of orbits responsible for the primary wing is comparable in number to the aligned population generating the secondary wing. Thus for uniform $M_{\mathrm{p}}$ the secondary wing does not outshine the primary as it does for uniform $f_{\mathrm{p}}$.

Varying the coefficient of gas $\operatorname{drag} A$ from $4 \times 10^{-4}$ to $4 \times 10^{-2} \mathrm{~s} / \mathrm{km}$, and likewise the number of ring crossings $\eta_{\text {kick }}$ from $10^{2}$ to $10^{4}$, yields sensible results. We achieve the same degree of apsidal alignment and the same images as long as $A \times \eta_{\text {kick }}>0.4 \mathrm{~s} / \mathrm{km}$, for $A \leq 0.04 \mathrm{~s} / \mathrm{km}$. For $A \geq 0.4 \mathrm{~s} / \mathrm{km}$-corresponding to $M_{\text {gas }} \geq 10^{-2} M_{\oplus}$ for our nominal set of parameters-velocity impulses at gas ring crossings are no longer perturbative (see equation 5). Such massive gas disks cannot be modeled using our zero-width approximation; they need to be spatially resolved and the trajectories of dust grains integrated in detail.

\section{SUMMARY AND DISCUSSION}

We have shown how an eccentric gas ring can strongly torque dust grain orbits in debris disks. If the gas ring does not eject a dust grain out of the system entirely, it forces the grain into apsidal alignment. That alignment produces disk 


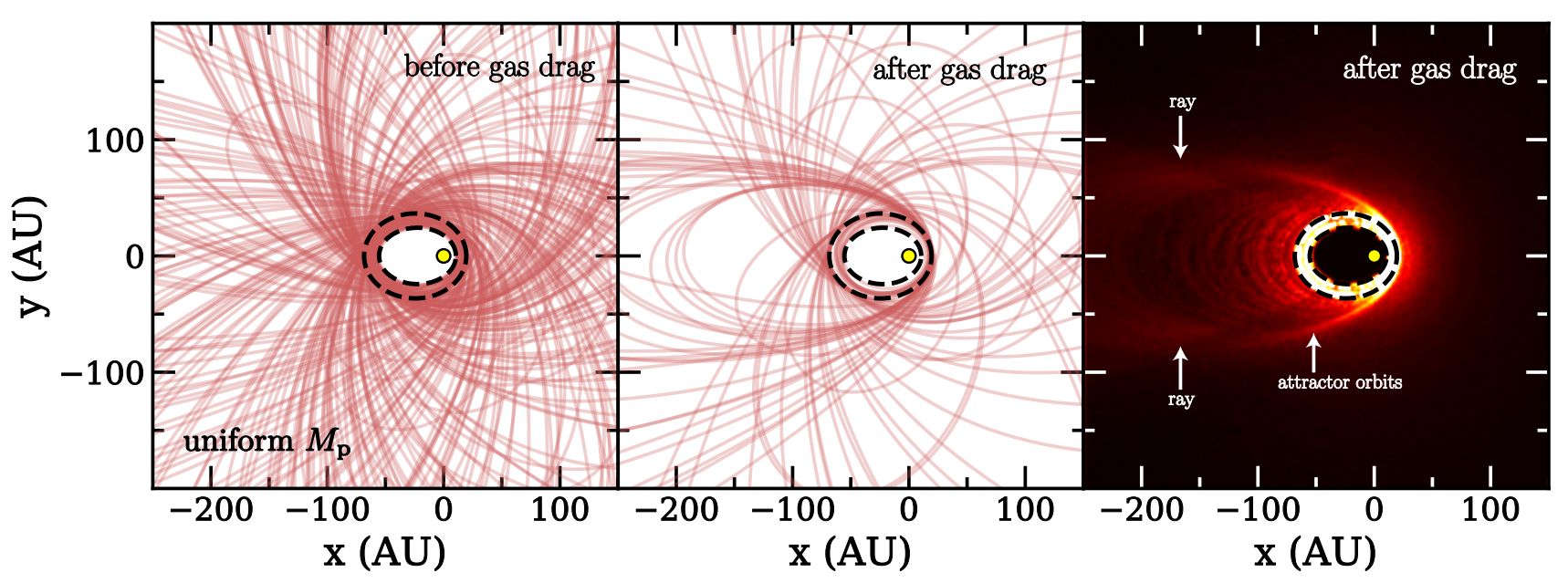

Figure 4. Top-down views of our standard model debris disk, before and after sculpting by an elliptical gas ring, for the case where dust grains are launched from a uniform distribution of parent body mean anomalies $M_{\mathrm{p}}$ (matching Figure 3). The parent body ring is bounded by the dashed black ellipses, and the idealized gas ring coincides with the outer black ellipse. The left panel plots a random sampling of dust grain orbits before gas drag, and the middle panel shows how those orbits (more precisely, those which remain bound, which are a subset) become more apsidally aligned after applying $\eta_{\text {kick }}=100$ gas drag impulses at fixed $A=0.04 \mathrm{~s} / \mathrm{km}$ (see equation 3 ). The right panel is a scattered light image (using the full set of $\sim 3 \times 10^{4}$ dust grain orbits) viewed top-down and smoothed with a 2D Gaussian of standard deviation 1 AU. In addition to the large "attractor orbits" whose periastra coincide with the gas ring and whose apastra grade smoothly outward, we see two "rays" emanating away from the parent body ring. These rays, when viewed nearly edge-on, comprise the secondary wing of the moth (Figure 3). The rays vary with intensity along their length; they are brightest in the region occupied by attractor orbits. See text for more discussion.

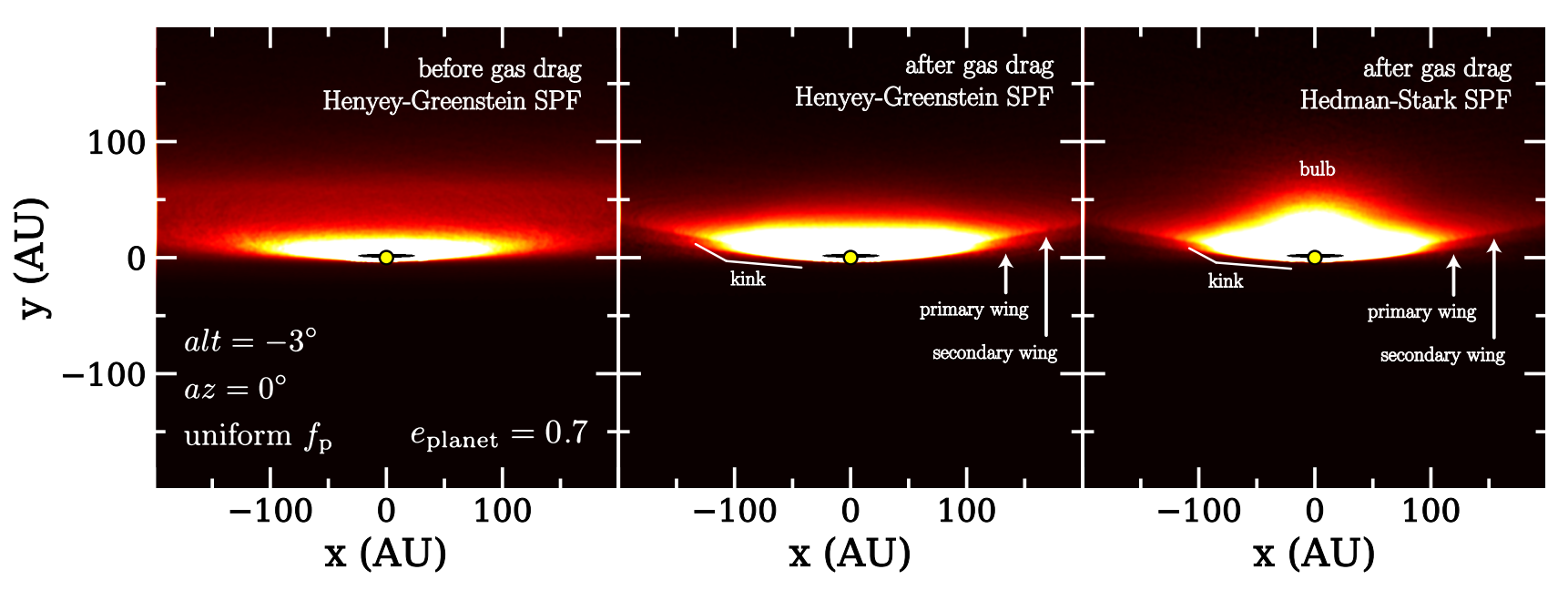

Figure 5. Same as Figure 3 but for the case where dust grain orbits are launched from a uniform distribution of parent body true anomalies $f_{\mathrm{p}}$. The secondary wing is more prominent than the primary wing because a greater proportion of dust orbits are on apsidally aligned orbits after gas drag (Figure 6).

morphologies in scattered starlight resembling "moths" having "double wings" that are "kinked" at their tips. These shapes recall those observed in HD 61005 and HD 32297 (e.g., Schneider et al. 2014; Esposito et al. 2016). The resemblance extends to their central "bulbs"- these are generated by grains at apse-aligned apastra, all pointing out of the sky plane at a small angle away from the line of sight-if we employ scattering phase functions appropriate to strongly forward-scattering, irregularly shaped particles (Hedman \& Stark 2015).

Our picture requires not only that debris disks like HD 61005 and HD 32297 have gas but that such gas have orbital 


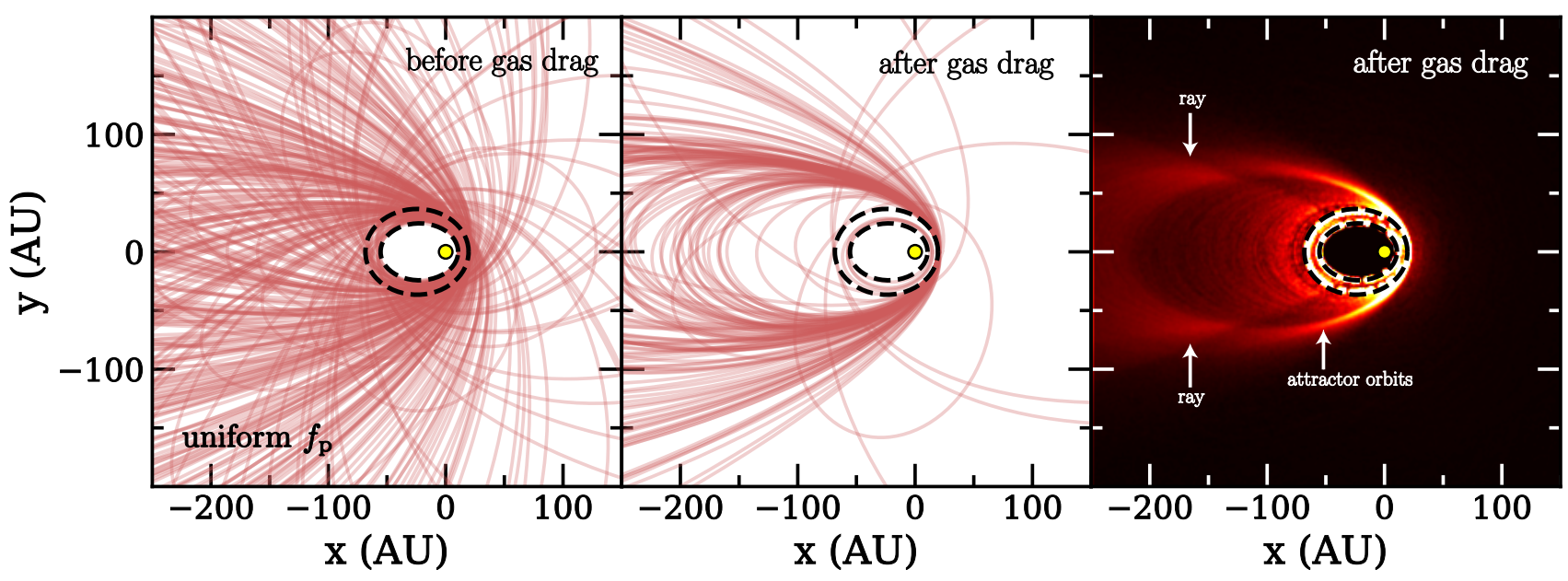

Figure 6. Same as Figure 4 but for the case where dust grain orbits are launched from a uniform distribution of parent body true anomalies $f_{\mathrm{p}}$. As compared to the uniform $M_{\mathrm{p}}$ case, here a greater fraction of dust orbits are apsidally aligned after gas drag, generating brighter rays and a brighter secondary wing relative to the primary (Figure 5).

eccentricities $\gtrsim 0.5$. The gas has insufficient mass for such large ellipticities to be caused by self-gravity. ${ }^{5}$ In our scenario, gas eccentricity derives instead from a perturber, itself on a highly eccentric orbit. The perturber should have a mass of at least a few Earths in order to gravitationally imprint its eccentricity on disk solids, and by extension the gas released from those solids, within the system age (e.g., Lee \& Chiang 2016).

Whether observations of gas in HD 61005 and HD 32297 are consistent with our theory is unclear. At least $\sim 10^{-3} M_{\oplus}$ of gas is needed to drag grains into alignment before they are collisionally destroyed ( $(2)$. To date no gas has been detected in HD 61005 (Olofsson et al. 2016; Riviere-Marichalar et al. 2016; MacGregor et al. 2018; §1) but an atomic disk of the necessary mass might have escaped detection. In addition to more sensitive observations, further modeling of the way that gas lines are excited in debris disks, in particular around this G8V star with a possible X-ray luminosity (Desidera et al. 2011), would be welcome. HD 32297 appears to have ample gas (MacGregor et al. 2018; Cataldi et al. 2019) though ironically probably too much for the calculations in this paper to apply: our method of idealizing the gas disk as a zero-width ring and applying small impulses to dust grain trajectories at discrete ring crossings prevents us from modeling gas disks more massive than $\sim 10^{-2} M_{\oplus}$, as these exert drag forces too large to be described as perturbative impulses. Thus the most pressing next step may be to spatially resolve the gas disk

\footnotetext{
${ }^{5}$ Even for eccentric gas modes which require, for host stellar mass $M_{*}$ and disk aspect ratio $h / r$, a gas mass of order $M_{*}(h / r)^{2}$, which is smaller than the Toomre mass $M_{*}(h / r)$. The former mass can be derived by balancing differential precession due to gas pressure gradients against differential precession by self-gravity.
}

(and its density, eccentricity, and/or apsidal gradients) and calculate dust trajectories under the dual influence of stellar radiation pressure and aerodynamic drag, to see whether massive gas disks of non-zero width can align grain apsides. Also worth assessing is whether an eccentricity can be detected in the gas orbiting HD 32297. This would manifest observationally not as an asymmetry between the two disk ansae (since we are viewing the system along its symmetry axis, i.e., the apsidal line) but rather as deviations from circular motion in the line-of-sight (radial) velocity of gas, measured against projected separation from the star. This would be a project for the Atacama Large Millimeter Array.

We have argued that gas, initially upon its release, traces out the same eccentric orbits occupied by the parent body ring $(\S 1)$. The long-term dynamical evolution of gas is less clear. How gas eccentricity is maintained or erased in the face of angular momentum transport by magnetorotational turbulence or magnetized winds (e.g., Kral \& Latter 2016) needs to be studied, together with eccentricity driving by a planet. It might be that only the gas closest to the parent body ring and the attendant planet is elliptical, and that gas circularizes as it diffuses radially from the source region. Or gas may be removed altogether by recondensation onto grains before diffusing very far (G. Cataldi 2019, personal communication).

In addition to turbulence, another threat to the coherence of the eccentric gas disk is drag back-reaction. We have considered throughout this paper how gas drags solids but neglected how solids drag gas. The back-reactive drag force scales as $f \propto \rho_{\mathrm{s}} / t_{\text {stop }}$, where $\rho_{s} \propto s^{1-q} s^{3}$ is the mass density of particles of size $s$ and $t_{\text {stop }} \propto s$ is the momentum stopping time of a particle in gas; this force tends to be dominated by small grains at the bottom of the cascade (e.g., $f \propto s^{-0.5}$ 
for a Dohnanyi differential size index $q=3.5$ ). As these small grains also feel radiation pressure and are apsidally misaligned at birth, they may disrupt an apsidally aligned gas disk if their mass exceeds that of gas. For bright debris disks like HD 61005 and HD 32297, the mass in small bound grains near the blow-out size is roughly $\sim 10^{-3} M_{\oplus}$ (see, e.g., equation 11 of Chiang et al. 2009). Thus back-reactive drag poses a concern for the models presented in this paper, which have a nominal gas mass of $M_{\text {gas }}=10^{-3} M_{\oplus}$. Whether this is just an order-unity detail or a showstopping issue needs to be worked out; regardless, back-reactive drag further motivates the study of more massive gas disks which cannot be treated in the zero-width limit but need to be spatially resolved.

Finally, are there alternatives to our proposal? We have explored here a quasi-steady scenario in which a ring of parent bodies, made eccentric by an eccentric planet, grinds down in a steady collisional cascade. But we can also consider a more stochastic picture. A single catastrophic disruption of a large body, occurring near periastron of its orbit and behind the host star relative to the observer, could spray dust onto the sought-for apsidally aligned orbits. This scenario would correspond to the "periapse only" model of Lee \& Chiang (2016). A recent outgassing has been invoked to explain the clumpy $\mathrm{CO}$ and $\mathrm{C}$ in $\beta$ Pic (Cataldi et al. 2018), and to explain the low C-to-CO abundance ratio in HD 32297 (Cataldi et al. 2019). Furthermore, the disks HD 61005 and
HD 32297 possess halos that are located hundreds of AUs from their host stars and are surprisingly bright in the millimeter continuum; the halos emit too strongly at long wavelengths to be interpreted in the usual way in terms of micronsized grains blown onto large orbits by radiation pressure (MacGregor et al. 2018). A catastrophic disruption could deliver millimeter-sized particles, produced either directly from the event or from subsequent collisional grinding of debris, to the large distances characterizing the halos (Y. Wu 2019, personal communication). Many elements of this alternative recent-disruption scenario remain to be quantified: the mass of the progenitor, the evolution of the size distribution of the debris, and the properties of the background disk from which the destroyed body was drawn.

We thank Eve Lee for assistance in navigating her debris disk code from which Figures 3-6 derive, and helpful feedback on the manuscript; Tom Esposito, Alexis Brandeker, Gianni Cataldi, Gaspard Duchêne, Matthew Hedman, Luca Matrà, and Yanqin Wu for insightful discussions and pointers to the wider literature that led to many changes in the paper; an anonymous referee for their thorough and thoughtful report that led to still more improvements, particularly with respect to observational searches for gas in HD 61005 and HD 32297; and Ian Czekala, Paul Kalas, Chris Stark, and David Wilner for encouraging exchanges. This work was supported by a Berkeley Excellence Account for Research.

\section{REFERENCES}

Brandeker, A., Cataldi, G., Olofsson, G., et al. 2016, A\&A, 591, A27

Buenzli, E., Thalmann, C., Vigan, A., et al. 2010, A\&A, 524, L1

Cataldi, G., Brandeker, A., Wu, Y., et al. 2018, ApJ, 861, 72

Cataldi, G., Wu, Y., Ohashi, N., et al. 2019, arXiv e-prints, arXiv:1904.07215

Cavallius, M., Cataldi, G., Brandeker, A., et al. 2019, arXiv e-prints, arXiv:1906.11106

Chen, C. H., Li, A., Bohac, C., et al. 2007, ApJ, 666, 466

Chiang, E., Kite, E., Kalas, P., Graham, J. R., \& Clampin, M. 2009, ApJ, 693, 734

Czechowski, A., \& Mann, I. 2007, ApJ, 660, 1541

Desidera, S., Covino, E., Messina, S., et al. 2011, A\&A, 529, A54

Esposito, T. M., Fitzgerald, M. P., Graham, J. R., et al. 2016, AJ, 152,85

Fernández, R., Brandeker, A., \& Wu, Y. 2006, ApJ, 643, 509

Grigorieva, A., Thébault, P., Artymowicz, P., \& Brandeker, A. 2007, A\&A, 475, 755

Hedman, M. M., \& Stark, C. C. 2015, ApJ, 811, 67

Hines, D. C., Schneider, G., Hollenbach, D., et al. 2007, ApJL, 671, L165
Kral, Q., \& Latter, H. 2016, MNRAS, 461, 1614

Kral, Q., Matrà, L., Wyatt, M. C., \& Kennedy, G. M. 2017, MNRAS, 469, 521

Kral, Q., Wyatt, M., Carswell, R. F., et al. 2016, MNRAS, 461, 845

Lee, E. J., \& Chiang, E. 2016, ApJ, 827, 125

MacGregor, M. A., Weinberger, A. J., Hughes, A. M., et al. 2018, ApJ, 869, 75

Maness, H. L., Kalas, P., Peek, K. M. G., et al. 2009, ApJ, 707, 1098

Matrà, L., Marino, S., Kennedy, G. M., et al. 2018, ApJ, 859, 72

Matrà, L., Öberg, K. I., Wilner, D. J., Olofsson, J., \& Bayo, A. 2019, AJ, 157, 117

Matrà, L., Panić, O., Wyatt, M. C., \& Dent, W. R. F. 2015, MNRAS, 447, 3936

Munson, B. R., Young, D. F., \& Okiishi, T. H. 2006, Fundamentals of Fluid Mechanics (J. Wiley \& Sons)

Olofsson, J., Samland, M., Avenhaus, H., et al. 2016, A\&A, 591, A108

Ricarte, A., Moldvai, N., Hughes, A. M., et al. 2013, ApJ, 774, 80

Riviere-Marichalar, P., Merín, B., Kamp, I., Eiroa, C., \&

Montesinos, B. 2016, A\&A, 594, A59 
Roberge, A., Feldman, P. D., Weinberger, A. J., Deleuil, M., \& Bouret, J.-C. 2006, Nature, 441, 724

Schneider, G., Grady, C. A., Hines, D. C., et al. 2014, AJ, 148, 59 